ACCEPTED MANUSCRIPT

\title{
On the electron density of atmospheric pressure radio frequency dielectric barrier discharge and discharge with bare electrode
}

To cite this article before publication: Lei Wang et al 2019 Jpn. J. Appl. Phys. in press https://doi.org/10.7567/1347-4065/ab5d74

\section{Manuscript version: Accepted Manuscript}

Accepted Manuscript is "the version of the article accepted for publication including all changes made as a result of the peer review process, and which may also include the addition to the article by IOP Publishing of a header, an article ID, a cover sheet and/or an 'Accepted Manuscript' watermark, but excluding any other editing, typesetting or other changes made by IOP Publishing and/or its licensors"

This Accepted Manuscript is @ 2019 The Japan Society of Applied Physics.

During the embargo period (the 12 month period from the publication of the Version of Record of this article), the Accepted Manuscript is fully protected by copyright and cannot be reused or reposted elsewhere.

As the Version of Record of this article is going to be / has been published on a subscription basis, this Accepted Manuscript is available for reuse under a CC BY-NC-ND 3.0 licence after the 12 month embargo period.

After the embargo period, everyone is permitted to use copy and redistribute this article for non-commercial purposes only, provided that they adhere to all the terms of the licence https://creativecommons.org/licences/by-nc-nd/3.0

Although reasonable endeavours have been taken to obtain all necessary permissions from third parties to include their copyrighted content within this article, their full citation and copyright line may not be present in this Accepted Manuscript version. Before using any content from this article, please refer to the Version of Record on IOPscience once published for full citation and copyright details, as permissions will likely be required. All third party content is fully copyright protected, unless specifically stated otherwise in the figure caption in the Version of Record.

View the article online for updates and enhancements. 


\title{
On the electron density of atmospheric pressure radio frequency dielectric barrier discharge and discharge with bare electrode
}

\author{
Lei Wang $^{1^{*}}$, Nikola Cvetanović ${ }^{2,3}$, Bratislav Obradović ${ }^{2}$, Eusebiu-Rosini Ionita ${ }^{4}$, Gheorghe \\ Dinescu $^{4}$, Christophe Leys ${ }^{1}$, Anton Nikiforov ${ }^{1 *}$ \\ ${ }^{1}$ Department of Applied Physics, Ghent University, Sint-Pietersnieuwstraat 41 B4, 9000 \\ Ghent, Belgium \\ ${ }^{2}$ Faculty of Physics, University of Belgrade, PO Box 368, 11001 Belgrade, Serbia \\ ${ }^{3}$ Faculty of Transport and Traffic Engineering, University of Belgrade, Vojvode Stepe 305, \\ 11000 Belgrade, Serbia \\ ${ }^{4}$ National Institute of Laser, Plasma and Radiation, Magurele-Bucharest, MG-36, Ilfov RO \\ 077125, Romania \\ *E-mail: lei.wang@ugent.be, anton.nikiforov@ugent.be
}

Atmospheric pressure radio frequency helium plasma with two different designs: dielectric barrier discharge (DBD) and discharge with bare electrode (DBE) were investigated by means of optical emission spectroscopy. Both DBD and DBE can work at relatively low temperature and produce abundant electrons facilitating production of reactive species through electron-impact reactions. Stark broadening method of Hydrogen Balmer beta $\left(\mathrm{H}_{\beta}\right)$ line was employed to analyze the electron density. When electron density is below $10^{20} \mathrm{~m}^{-3}$, fine-structure fitting was used to improve the accuracy of electron density estimation. At power ranged 4-20 W, DBD and DBE showed electron density 4.1-6.1 $\times 10^{19} \mathrm{~m}^{-3}$, and 3.6$8.6 \times 10^{19} \mathrm{~m}^{-3}$, respectively. The DBD is more suitable than DBE for biomedical applications due to the wider working power range and lower gas temperature in the range of 316-344 K, depending on the power.

\section{Introduction}

In the recent decade, atmospheric pressure plasma has been a hot research topic for biomedical applications such as medicine and hygiene ${ }^{1-4)}$, due to its capability to produce abundant reactive Oxygen/Nitrogen species (RONS) ${ }^{5-7)}$. Generally, the atmospheric pressure plasmas can be categorized according to the electrical excitation frequency including direct current (DC) plasma, alternating current (AC) plasma, radio-frequency (RF) plasma, microwave plasma ${ }^{8)}$. Among these types of plasma, radio frequency (RF) plasmas are excellent for those applications due to advantages of relatively low cost and electrical safety, 
capability of treatment of non-conductive objects ${ }^{8,9)}$. Generally speaking, relatively low temperature and sufficient RONS are required in the clinical fields ${ }^{10-12)}$. The RONS are produced during the transportation of reactive species (electrons, ions, excited species, radicals) from plasma region down to the treated sample ${ }^{5,13)}$. These reactive species are highly related to the electron-impact reactions ${ }^{10,14)}$. Therefore, electron density investigations are needed to understand and enhance the production of reactive species.

There are several kinds of methods to measure the electron density in plasma, such as Stark broadening method ${ }^{15,16)}$, Thompson scattering method ${ }^{17,18)}$, etc. Stark broadening method with $\mathrm{H}_{\beta}$ line based on optical emission spectroscopy (OES) shows advantages of simple installation and alignment. Thus it is widely applied for electron density measurements. Electron density measurements were performed by many researchers in atmospheric pressure helium discharge under direct current or alternated current (AC) electrical conditions. Ionascut-Nedelcescu et al. analyzed electron density to be less than 4 $\times 10^{18} \mathrm{~m}^{-3}$ at voltage of $10.8 \mathrm{kV}$ in an AC (25 kHz) atmospheric pressure dielectric barrier helium discharge torch ${ }^{19}$. Takeda et al. calculated the electron density in two glow-like plasmas to be $9 \times 10^{19} \mathrm{~m}^{-3}$ in an AC $(50 \mathrm{~Hz})$ streamer-like helium discharge at a peak-topeak voltage of $9 \mathrm{kV}$ and $1.2 \times 10^{20} \mathrm{~m}^{-3}$ in an AC (62 kHz) dielectric barrier helium discharge at a peak-to-peak voltage of $6 \mathrm{kV}^{20)}$. Hoffmann et al. obtained electron density to be $(3.4 \pm$ 2.5) $\times 10^{19} \mathrm{~m}^{-3}$ for dissipated power of $0.1-12 \mathrm{~W}$ in atmospheric pressure helium RF (11.7 $\mathrm{MHz}$ ) discharge in the diffuse mode between a tungsten needle and grounded plate electrode $^{21)}$. However, the amount of experiments and data on atmospheric pressure RF plasma sources are still quite limited in the literature. Also, it is expected that electron densities are dependent upon discharge configurations and power ranges.

In this study, we investigated and compared atmospheric pressure RF DBD and DBE in terms of electrical characteristics, gas temperature, and electron density. The DBD and DBE have the same size and structure of the components for the electrode and the dielectric, while their discharge configurations are distinguished based on whether plasma has direct contact with the electrodes. This work is aiming to contribute to the design optimization of atmospheric pressure plașma by comparing both DBD and DBE, as well as to the investigations of electron density for low temperature RF plasma sources.

\section{Experimental methods}

The geometry of the two atmospheric pressure helium plasma sources and optical emission spectroscopy diagnostics setup are depicted in Fig. 1. Figure 1(a) shows the RF DBD with 
double dielectric layers embedded into powered electrode and grounded electrode as interiors; Figure 1(b) presents the RF DBE where electrodes were rotated $180^{\circ}$ and no dielectric layers were in contact with plasma. The dielectric layer is made of $99 \%$ alumina $\left(\mathrm{Al}_{2} \mathrm{O}_{3}\right)$. In DBE configuration, the power and ground electrodes were directly connected to the terminals. Both plasma sources shared the same dimensions for manufacturing engineering and working conditions including input power, gas type, flow rate, in order to see the geometric structure influence on the discharge properties. The plasma source consisted of two trapezoidal ceramic dielectric layers with $0.6 \mathrm{~mm}$ width and $8 \mathrm{~mm}$ height. The discharge gap has a height of $1 \mathrm{~mm}$, which is fixed by two ceramic spacers. As seen in Fig. 1(c) for layout of experiment setup with diagnostic system, helium gas was fed into the gap at gas flow rate of 2 SLM controlled by a mass flow controller (Bronkhorst EL-FLOW). An RF power generator (ADTEC AX-600III-A-NV1) combined with an L type automatic impedance matching network (ADTEC AMV-1000-EN) empowered the helium discharge, whose voltage and current was further recorded by an oscilloscope (TEKTRONIX MSO5104B) with $1 \mathrm{GHz}$ bandwidth (10 GS/s sample rate) through a Vigilant Sensor RF voltage-current probe. The RF probe was placed between plasma source and matching network. After ignition and stabilization of the discharge, the plasma effluent of about $5 \mathrm{~mm}$ length was observed in the surrounding air. A CCD camera (Andor iDus 420-OE) was located at the exit slit of a spectrometer (HORIBA FHR 1000) and captured the discharge emission that was projected with a focus lens into the entrance slit (width of $12 \mu \mathrm{m}$ ) of the Horiba spectrometer. To be more specific, the CCD camera conducted accumulations for each time integrated spectrum acquisition and Horiba spectrometer used the 2400 grooves $/ \mathrm{mm}$. The lens was placed away $13 \mathrm{~cm}$ from the plasma source and $7 \mathrm{~cm}$ from the spectrometer. The relative humidity in ambient air during experiments was around $60 \%$.

The total power is indicated by the power generator and the dissipated power is calculated based on voltage, current, and phase shift recorded by the oscilloscope. A low-pressure mercury lamp (Pen-Ray) was used to measure the instrumental function of the spectrometer at wavelength of $435.83 \mathrm{~nm}$. This wavelength was chosen because it approximates the wavelength of $\mathrm{H}_{\beta}$ line which was used for electron density analysis in this work.

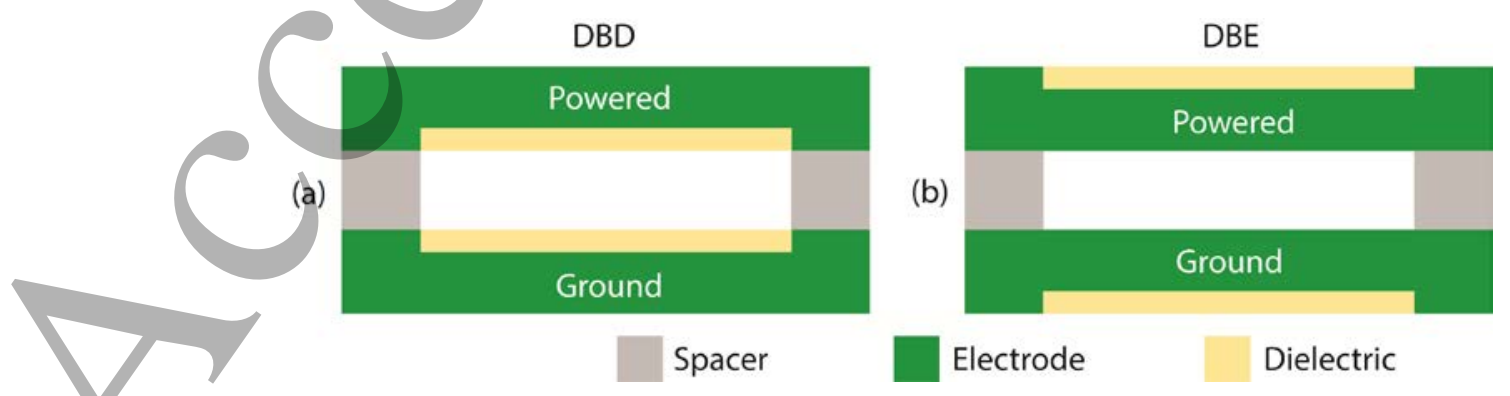


(c)

\section{Results and discussion}

\subsection{Voltage and current characteristics}

Atmospheric pressure plasmas used for bio-applications are required to exhibit the mild temperature for tissue or cells and abundant active species, which are related to working modes. A proper working mode of plasma enables realizing its full potential for bioapplications and was characterized by electrical measurements in this study. The electrical characteristics of DBD are presented in Fig. 2(a). The DBD discharge was ignited as $\alpha$ mode homogeneous discharge at input power of $6 \mathrm{~W}$, voltage $155 \mathrm{~V}$, and can be sustained at power as low as $4 \mathrm{~W}$ (voltage $140 \mathrm{~V}$ ). When increasing input power, the voltage and current also rose until transiting to $\gamma$ mode above the power of $36 \mathrm{~W}$ (voltage $335 \mathrm{~V}$ ). The phase shift magnitude of $\alpha$ mode was in the range of $89.0^{\circ}$ and $85.5^{\circ}$, showing capacitive properties and a decreasing trend with the increase of input power because gas ionization became more significant. Similar phenomena were also observed for DBE (see Fig. 2(b)). The DBE can be ignited as a mode at power of $5 \mathrm{~W}$ (voltage $134 \mathrm{~V}$ ) and sustained at low power of $4 \mathrm{~W}$ (voltage $127 \mathrm{~V}$ ). The voltage and current also became higher with the increment of input power, until the discharge reaching $\gamma$ mode at the power of $24 \mathrm{~W}$ (voltage $245 \mathrm{~V}$ ). The phase shift ranged from $88.8^{\circ}$ to $84.4^{\circ}$ and declines with increasing power. The voltage and current 
waveforms in the $\alpha$ mode of both DBD and DBE showed sinus waveforms with negligible harmonics. The ignition power and voltage of DBD was a little higher than those of DBE, probably due to the charge accumulation on dielectric layer inhibiting the formation of discharge. The constricted $\gamma$ mode is generally avoided for biomedical application due to the localized treatment area and high gas temperature ${ }^{22)}$. Therefore, the DBD can work in a larger power scale of $4 \mathrm{~W}$ to $36 \mathrm{~W}$, while DBE was able to operate in the power range of 4 $\mathrm{W}$ to $24 \mathrm{~W}$. The direct contact between discharge with metal electrode in DBE is responsible for the easy transition to $\gamma$ mode. The phase shift of DBD at the same input power is shightly higher than DBE, which can be attributed to the capacitive function of dielectric layer in DBD. It is also noticed that the dissipated power of $\alpha$ mode in DBE is also higher than that in DBD at the same input power, which makes DBE a more efficient plasma source.
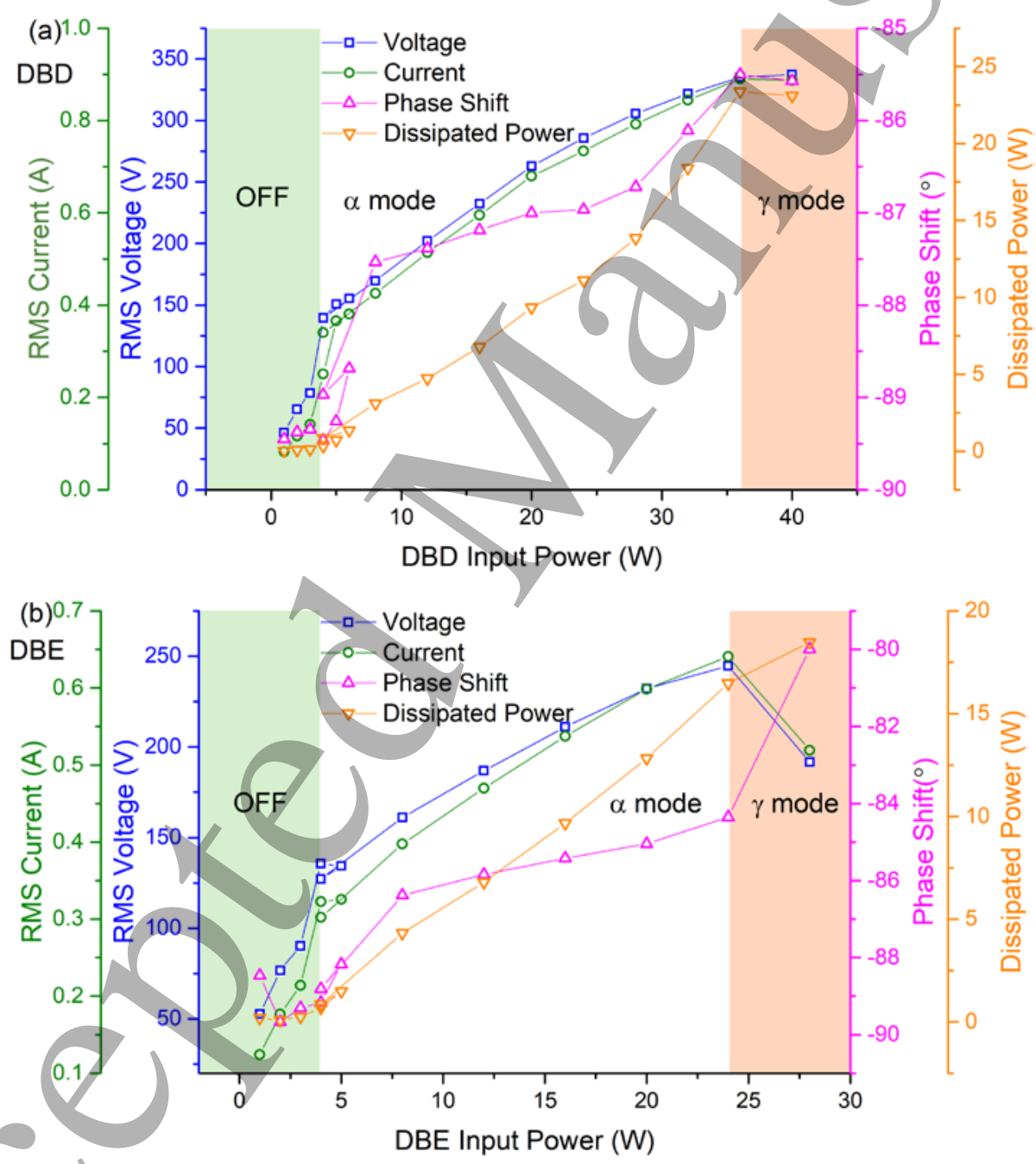

Fig. 2. Voltage, current, phase shift, dissipated power variation with input power at helium flow rate of 2 SLM for (a) DBD and (b) DBE.

3.2 Optical emission spectroscopy 
Optical emission spectroscopy was employed to record the discharge emission spectrum to obtain the gas temperature and electron density, and to identify the produced species. Figure 3 shows the emission spectrum of DBD at power of $20 \mathrm{~W}$. Many molecular bands and atomic lines are observed including $\mathrm{NO}(\mathrm{A}-\mathrm{X})$ bands, $\mathrm{OH}(\mathrm{A}-\mathrm{X})$ bands, $\mathrm{N}_{2}(\mathrm{C}-\mathrm{B})$ bands, $\mathrm{N}_{2}{ }^{+}(\mathrm{B}-\mathrm{X})$ bands, $\mathrm{He} \mathrm{I}$ lines, $\mathrm{H}_{\alpha}$ line, $\mathrm{H}_{\beta}$ line, $\mathrm{N}$ I line, $\mathrm{O}$ I lines. He I lines can be assigned to helium excited states, while other bands and lines are due to the impurities of feeding gas (mainly $\mathrm{H}_{2} \mathrm{O}$ vapor) and diffusion of humid ambient air $\left(\mathrm{N}_{2}, \mathrm{O}_{2}, \mathrm{H}_{2} \mathrm{O}\right)$ in the discharge region and effluent. The emission spectrum of DBE is also similar to DBD, thus not presented here for simplification. The emission spectrum can also reveal the chemically rich environment in both discharges for bio-applications.

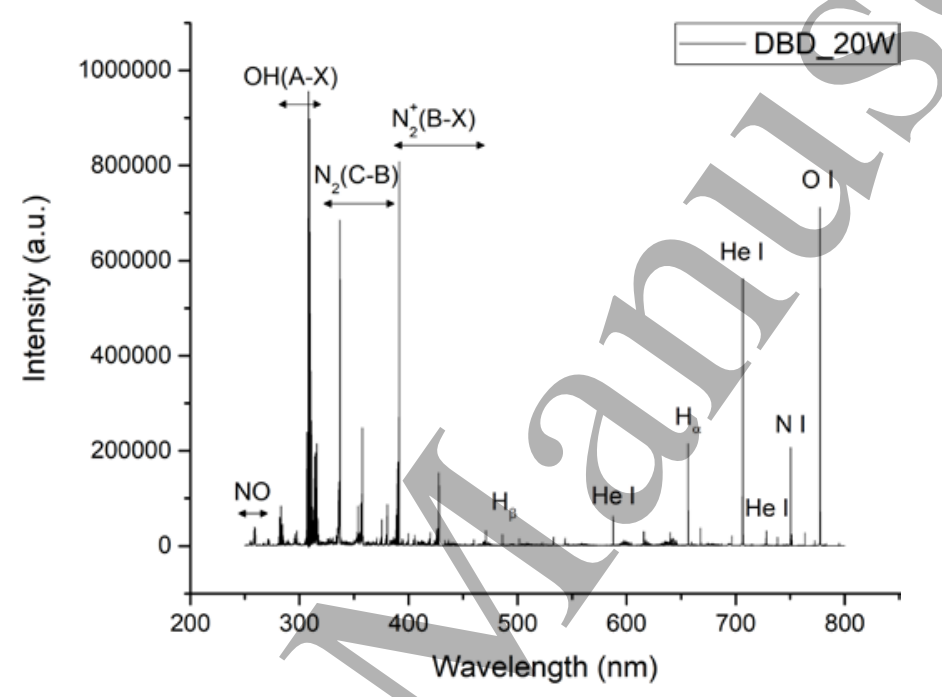

Fig. 3. Emission spectrum in the range of $200-800 \mathrm{~nm}$ for DBD at the power of $20 \mathrm{~W}$.

In order to have better comparison for $\mathrm{DBD}$ and $\mathrm{DBE}$, the experiment data were collected under the same input power from $4 \mathrm{~W}$ to $20 \mathrm{~W}$ in the $\alpha$ mode. Gas temperature was estimated by measuring the rotational temperature of $\mathrm{OH}(\mathrm{A})$ excited states-a method commonly used in low temperature plasma ${ }^{23-26)}$. The $\mathrm{OH}\left(A^{2} \Sigma^{+} \rightarrow X^{2} \Pi, 0-0\right)$ bands in the range of 306-312 $\mathrm{nm}$ is recorded and fitted using massiveOES software ${ }^{27)}$. This software was described and successfully applied to calculate the gas temperature in previous research ${ }^{26,28)}$. Figure 4(a) gives an example of the fitting $\mathrm{OH}$ bands of $\mathrm{DBD}$ at power of $20 \mathrm{~W}$ with an inset of Boltzmann plot confirming the estimation of rotational temperature. The Boltzmann distribution was obtained for rotational quantum $\mathrm{J}$ number less than 8 and results showed a rotational temperature around $344 \mathrm{~K}$. The estimated temperature has an error of $25 \mathrm{~K}$ from methodology. The fitting procedures are repeated for $\mathrm{OH}$ bands acquired at other input power for DBD and DBE to estimate their gas temperatures presented in Fig. 4(b). As expected, the gas temperature increases with input power for both plasma sources. DBD exhibited the 
temperature in the range of $(316 \pm 25) \mathrm{K}$ to $(344 \pm 25) \mathrm{K}$, which was lower than the gas temperature of DBE in the range of $(319 \pm 25) \mathrm{K}$ to $(361 \pm 25) \mathrm{K}$ at the same input power. This phenomenon is also related to the dielectric layer that prevents the discharge from being too intensive. Due to the relatively low gas temperature, both plasma sources are able to be operated for biomedical applications.
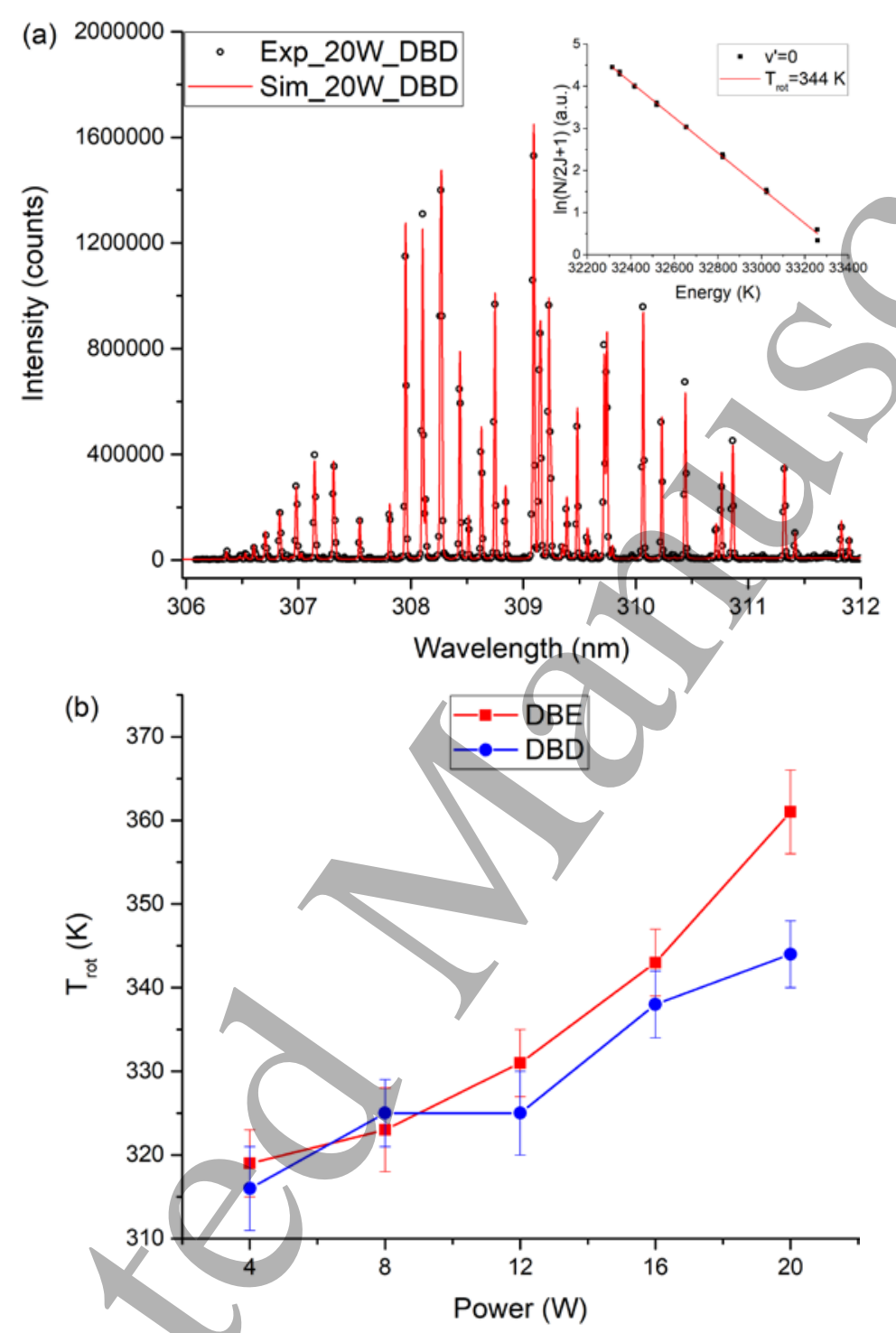

Fig. 4. Gas temperature estimation: (a) OH bands fitting with massiveOES; (b) Gas temperature for DBD and DBE at different input power and fixed helium flow rate of 2 SLM.

\subsection{Electron density}

The reactive species formation for biomedical applications is highly related to the electroncollision reactions. Here the $\mathrm{H}_{\beta}$ line is used to obtain electron density due to its advantages of strong Stark broadening dependence on electron density, and weak sensitivity to electron temperature ${ }^{29)}$. However, it also has a disadvantage that this method can only be applied in 
the case of considerably high electron density $\left(>10^{20} \mathrm{~m}^{-3}\right)$ making it difficult to apply to many types of low temperature plasmas. In this study, we first use the classical Voigt fitting method to analyze the $\mathrm{H}_{\beta}$ line profiles to obtain the electron density. After that, calculated electron density was found to be below the threshold of $10^{20} \mathrm{~m}^{-3}$, which may lead to significant error and uncertainty. A fine-structure fitting method was then applied to get more accurate results. This fitting method is based on assuming that the broadening mechanism of $\mathrm{H}_{\beta}$ line also applies to the 7 fine-structure components ${ }^{29,30}$. This method has been used by Hoffman et al. ${ }^{21)}$ to analyze the electron density in atmospheric pressure RF plasma jet. Palomares et al. ${ }^{29)}$ experimentally validated the applicable range of this method down to $6 \times 10^{18} \mathrm{~m}^{-3}$ by comparing with a different, independent method-Thomson scattering.

The line profile is broadened by different mechanisms: instrumental broadening, natural broadening, self-absorption, Doppler broadening, Van der Waals broadening, resonance broadening, and Stark broadening. The instrumental broadening in our case has a Gaussian profile and full width at half maximum (FWHM) of $0.014 \mathrm{~nm}$. The natural broadening is negligible compared to other broadenings ${ }^{31)}$. Self-absorption can be presumed as nonsignificant for $\mathrm{H}_{\beta}$ line in non-hydrogen low temperature plasma ${ }^{16)}$. Doppler contribution to line profile has a Gaussian shape ${ }^{32)}$ and is calculated with the gas temperature obtained from Sect. 3.2. The same gas temperature was used for the Van der Waals broadening, which is caused by interaction between excited atoms and surrounding neutral particles and has a Lorentzian profile ${ }^{32)}$.

Resonance broadening occurs when lower states are dipole coupled to ground state and strongly depends on the density of ground states of the same emitters ${ }^{32)}$. In the present case, the relative density of the $\mathrm{H}$ atoms is low because helium serves as the working gas. Therefore, resonance broadening of $\mathrm{H}_{\beta}$ line can be negligible.

On one hand, in the classical Voigt fitting, the $\mathrm{H}_{\beta}$ line is considered as a single line profile function and Stark contribution is assumed to be Lorentzian. The fitting is then achieved by convoluting Gaussian instrumental and Doppler broadening, and Lorentzian Van der Waals and Stark broadening. On the other hand, in the fine-structure fitting, the Stark broadening is analyzed by assigning a Voigt shape to each fine component. The overall profile is then analyzed by convoluting separate fine structure transitions of $\mathrm{H}_{\beta}$ as line profiles, with relative line strengths and line shifts being the same as in the zero-field case ${ }^{30)}$. Due to the low plasma density, this may be taken as a valid assumption. In other words, each fine-structure transition is assigned with its corresponding Doppler, Van der Waals, Stark broadening, and instrumental broadening so that the overall profile is obtained as a superposition of profiles. 
In this approach, the same Stark width which is expected from theory for the entire line, is assigned to each fine-structure component. The fine-structure fitting can be used to improve the accuracy of electron density calculation close to the threshold of classical method, but above all for checking the validity of the obtained results for plasma density in the conditions when Van der Waals broadening is below the fine-structure limit of $0.05 \mathrm{~nm}^{15,16)}$.

The electron densities measured with both fitting methods for DBD and DBE under different powers are shown in Fig. 5. Figure 5(a) shows the fitting profile of $\mathrm{H}_{\beta}$ line for $\mathrm{DBD}$ at 20W. It can be seen that Voigt fitting profile and fine-structure fitting profile overlap due to the relatively small discrepancy. The electron density obtained from Voigt fitting is $6.5 \times$ $10^{19} \mathrm{~m}^{-3}$, which is a little lower than the electron density derived from fine-structure fitting showing $(6.1 \pm 0.8) \times 10^{19} \mathrm{~m}^{-3}$. When using of $\mathrm{H}_{\beta}$ line Stark broadening method for electron density close to $1 \times 10^{20} \mathrm{~m}^{-3}$, but still above the lower $\operatorname{limit}\left(4 \times 10^{19} \mathrm{~m}^{-3}\right)^{33)}$, the finestructure effect contributes to a small extent to the final results. Correspondingly, the electron density can be estimated by use of simple Voigt fitting with acceptable accuracy.
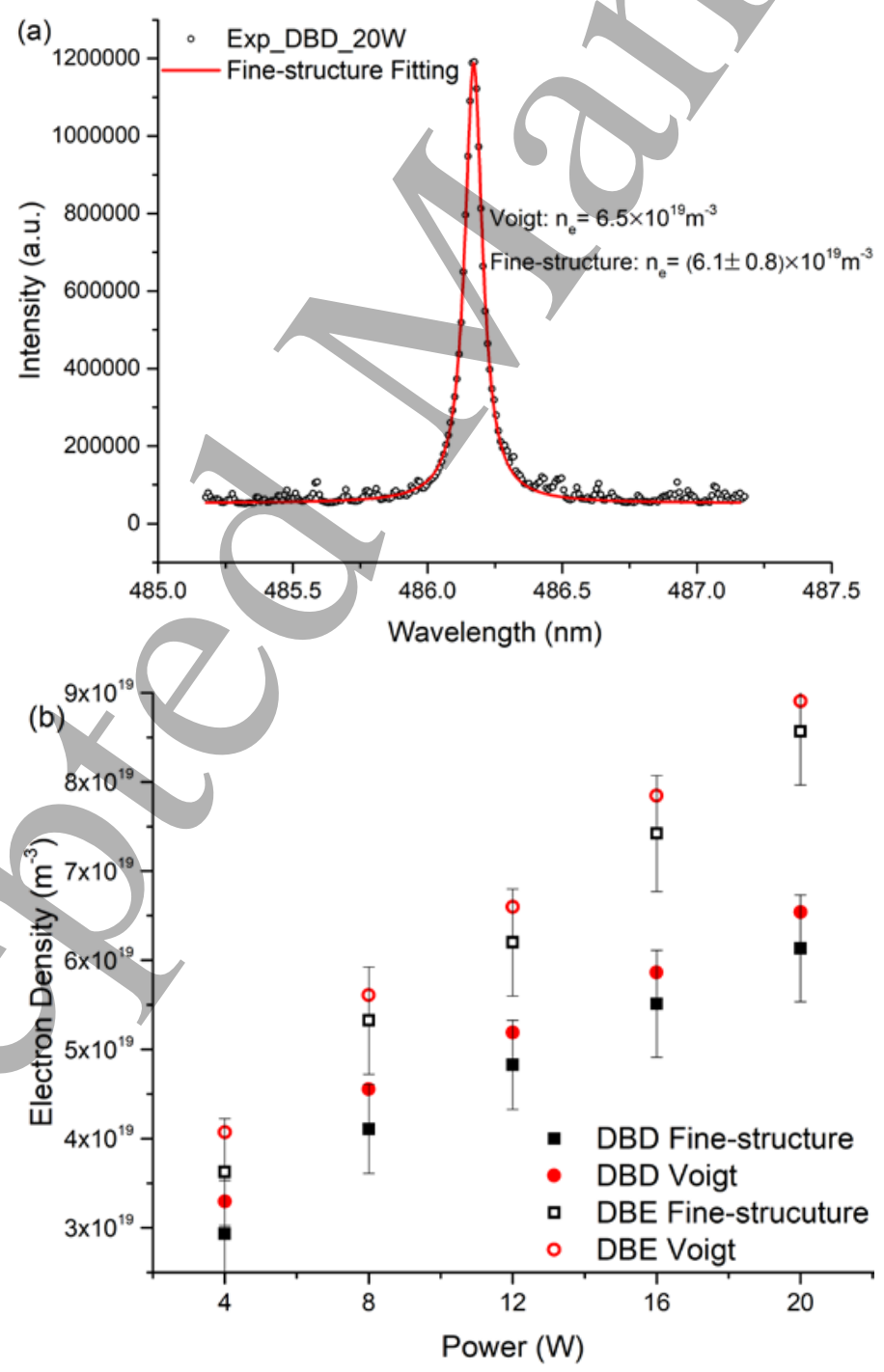
Fig. 5. Electron density obtained by Stark broadening method: (a) fitting of $\mathrm{H}_{\beta}$ line. Voigt fitting and fine-structure fitting overlap; (b) electron density of both DBD and DBE at different powers.

Figure 5(b) shows that the electron density obtained from Voigt fitting is also lower than that from fine-structure fitting for both DBD and DBE. When applying the power from $4 \mathrm{~W}$ to $20 \mathrm{~W}$, the DBD and DBE have the electron density in the range from $4.1 \times 10^{19} \mathrm{~m}^{-3}$ to 6.1 $\times 10^{19} \mathrm{~m}^{-3}$, and from $3.6 \times 10^{19} \mathrm{~m}^{-3}$ to $8.6 \times 10^{19} \mathrm{~m}^{-3}$, respectively. These electron density results are similar to those obtained by Hofmann et. $a l^{20,21)}$ investigating the atmospheric pressure RF helium plasma jet. Higher electron density measured in DBE discharge than in $\mathrm{DBD}$ at the same input RF power is probably explained by presence of the dielectric layer. The layer results in higher capacitance of the gap and thus decreases dissipated power; however, it makes the discharge more stable and suppresses transition to the $\gamma$ mode. It should also be emphasized that in the experiment, the overall emission from discharge zone and the plume are projected into the entrance slit of spectrometer and recorded by 1000 accumulations for each gate time of $0.5 \mathrm{~s}$. The electron density measured here by $\mathrm{H}_{\beta}$ line analysis lacks spatial and temporal resolution and is predominantly characterized by the maximum electron density. This phenomenon has also been observed in the previous experiment $^{20)}$. Although higher electron density was found in DBE than in DBD, it has to be emphasized that DBD configuration is more stable and $\alpha$ mode homogeneous plasma can be sustained at higher power than DBE, which makes DBD configuration of the RF plasmas favorable for biomedical applications.

\section{Conclusions}

Aiming at bio-applications, atmospheric pressure RF DBD and DBE are investigated regarding the electrical characteristics, gas temperature, as well as the electron density. Both DBD and DBE operate as the capacitive discharge, and can work at relatively low temperature, and are expected to produce abundant species due to electron-collision reactions for biomedical applications. However, there are some differences for both plasma sources due to the presence of dielectric barrier layers in DBD. DBD shows a larger working power range and slightly lower gas temperature at the same input power. Although DBE shows higher electron density than DBD at the same power, DBD can be sustained in the stable and homogeneous $\alpha$ mode at higher power to achieve a similar magnitude of electron density to promote chemistry activities. Therefore, atmospheric pressure RF DBD design is more desirable for bio-applications. 


\section{Acknowledgments}

This work was supported by the China Scholarship Council (File No. 201503170253) and co-funding of Ghent University (Reference Code: DOZA/DDC/AM/006b-2016). The work was partially supported by the M Era-Net program, project 'PlasmaTex'. This work within the Project 141043 was also supported by the Ministry of Science of the Republic of Serbia.

\section{References}

1) S. Bekeschus, P. Favia, E. Robert, and T. von Woedtke, Plasma Process. Polym. 16, 1800033 (2019).

2) H. Tanaka, K. Ishikawa, M. Mizuno, S. Toyokuni, H. Kajiyama, F. Kikkawa, H.-R. Metelmann, and M. Hori, Rev. Mod. Plasma Phys. 1, 3 (2017).

3) T. Homma, M. Furuta, and Y. Takemura, Jpn. J. Appl. Phys. 52, 036201 (2013).

4) T. Hirata, T. Kishimoto, C. Tsutsui, T. Kanai, and A. Mori, Jpn. J. Appl. Phys. 53, 010302 (2014).

5) N.Y. Babaeva and G. V. Naidis, Trends Biotechnol. 36, 603 (2018),

6) D.B. Graves, J. Phys. D. Appl. Phys. 45, 263001 (2012).

7) S.J. Kim and T.H. Chung, Sci. Rep. 6, 20332 (2016).

8) J. Winter, R. Brandenburg, and K.-D. Weltmann, Plasma Sources Sci. Technol. 24, 064001 (2015).

9) S. Reuter, T. von Woedtke, and K.-D. Weltmann, J. Phys. D. Appl. Phys. 51, 233001 (2018).

10) X. Lu, G.V. Naidis, M. Laroussi, S. Reuter, D.B. Graves, and K. Ostrikov, Phys. Rep. 630, 1 (2016).

11) K.-D. Weltmann and T. von Woedtke, Eur. Phys. J. Appl. Phys. 55, 13807 (2011).

12) K.-D. Weltmann and T. von Woedtke, Plasma Phys. Control. Fusion 59, 014031 (2017).

13) S.A. Norberg, E. Johnsen, and M.J. Kushner, Plasma Sources Sci. Technol. 24, 035026 (2015).

14) D.X. Liu, P. Bruggeman, F. Iza, M.Z. Rong, and M.G. Kong, Plasma Sources Sci. Technol. 19, 025018 (2010).

15) N. Konjević, M. Ivković, and N. Sakan, Spectrochim. Acta - Part B At. Spectrosc. 76, 16 (2012).

16) A.Y. Nikiforov, C. Leys, M.A. Gonzalez, and J.L. Walsh, Plasma Sources Sci. Technol. 24, 034001 (2015).

17) S. Hübner, J.S. Sousa, V. Puech, G.M.W. Kroesen, and N. Sadeghi, J. Phys. D. Appl. Phys. 47, 432001 (2014).

18) S.G. Belostotskiy, R. Khandelwal, Q. Wang, V.M. Donnelly, D.J. Economou, and N. Sadeghi, Appl. Phys. Lett. 92, 221507 (2008).

19) A. Ionascut-Nedelcescu, C. Carlone, U. Kogelschatz, D. V. Gravelle, and M.I. Boulos, J. Appl. Phys. 103, (2008).

20) K. Takeda, H. Yamada, K. Ishikawa, H. Sakakita, J. Kim, M. Ueda, J. Ikeda, Y. Akimoto, Y. 
Kataoka, N. Yokoyama, Y. Ikehara, and M. Hori, J. Phys. D. Appl. Phys. 52, 165202 (2019).

21) S. Hofmann, A.F.H. van Gessel, T. Verreycken, and P. Bruggeman, Plasma Sources Sci. Technol. 20, 065010 (2011).

22) X. Yang, M. Moravej, G.R. Nowling, S.E. Babayan, J. Panelon, J.P. Chang, and R.F. Hicks, Plasma Sources Sci. Technol. 14, 314 (2005).

23) L. Wang, G. Dinescu, X. Deng, E.-R. Ionita, C. Leys, and A.Y. Nikiforov, Plasma Sources Sci. Technol. 26, 075012 (2017).

24) P.J. Bruggeman, N. Sadeghi, D.C. Schram, and V. Linss, Plasma Sources Sci. Technol. 23, 023001 (2014).

25) K.C. Hsieh, H. Wang, and B.R. Locke, Plasma Process. Polym. 13, 908 (2016).

26) J. Voráč, P. Synek, V. Procházka, and T. Hoder, J. Phys. D. Appl. Phys. 50, 294002 (2017).

27) J. Voráč and P. Synek, Https://Bitbucket.Org/OES_muni/Massiveoes (2016).

28) J. Voráč, P. Synek, L. Potočňáková, J. Hnilica, and V. Kudrle, Plasma Sources Sci. Technol. 26, 025010 (2017).

29) J.M. Palomares, S. Hübner, E.A.D. Carbone, N. de Vries, E.M. van Veldhuizen, A. Sola, A. Gamero, and J.J.A.M. van der Mullen, Spectrochim. Acta Part B At. Spectrosc. 73, 39 (2012).

30) J. Reader, Appl. Spectrosc. 58, 1469 (2004).

31) Q. Xiong, A.Y. Nikiforov, M.Á. González, C. Leys, and X.P. Lu, Plasma Sources Sci. Technol. 22, 015011 (2012).

32) T. Belmonte, C. Noël, T. Gries, J. Martin, and G. Henrion, Plasma Sources Sci. Technol. 24, 064003 (2015).

33) B. Grabowski, W. Olchawa, and R. Olchawa, Eur, Phys. J. D - At. Mol. Opt. Phys. 28, 119 (2004).

\section{Figure Captions}

Fig. 1. Geometry of the two atmospheric pressure RF helium plasma sources and diagram of the experimental setup: (a) DBD; (b) DBE; (c) experimental setup with diagnostic system. The voltage is applied directly on powered electrode and dielectric layers in DBE are used only to keep the same structure for manufacturing as in DBD.

Fig. 2. Voltage, current, phase shift variation, dissipated power with input power at helium flow rate of 2 SLM for (a) DBD and (b) DBE.

Fig. 3. Emission spectrum in the range of 200-800 $\mathrm{nm}$ for DBD at the power of $20 \mathrm{~W}$.

Fig. 4. Gas temperature estimation: (a) $\mathrm{OH}$ bands fitting with massiveOES; (b) Gas temperature for DBD and DBE at different input power and fixed helium flow rate of 2 SLM. Fig. 5. Electron density obtained by Stark broadening method: (a) fitting of $\mathrm{H}_{\beta}$ line. Voigt fitting and fine-structure fitting overlap; (b) electron density of both DBD and DBE at 
different powers. 